\section{Urolithiasis mit Infekt ist überwiegend Frauensache}

Obwohl Männer deutlich öfter Harnsteine entwickeln als Frauen, tritt eine Urolithiasis mit Infektion bei Frauen zweimal häufiger auf. Dies hat eine Studie aus den USA gezeigt - und auch, dass die Inzidenz des Leidens insgesamt zunimmt.

$U_{\text {s.t. }}$ S-amerikanische Urologen haben im Zuge einer Studie die Trends beschrieben, die in den Jahren 1999 bis 2009 in der Inzidenz, dem praktischen Vorgehen und den Therapieergebnissen bei Steinen des oberen Harntraktes mit begleitenden Infektionen zu verzeichnen waren. Sie bedienten sich dabei eines USweiten Registers mit Daten von stationär behandelten Patienten. Für den besagten Zeitraum waren dort knapp 400.000 Fälle gelistet, die als infizierte Urolithiasis diagnostiziert worden waren.

Dabei stellte sich heraus, dass die entsprechende Inzidenz bei Frauen von $15,5 / 100.000$ im Jahr 1999 auf 27,6/100.000 im Jahr 2009 gestiegen war. Auch bei Männern gab es einen Zuwachs, und zwar von 7,8 auf $12,1 / 100.000$.
Gleichzeitig sind die Frauen aber in der Minderheit, was Steinleiden insgesamt betrifft; das Verhältnis liegt hier laut Schätzungen bei 68,4 vs. $105,0 / 100.000$ zu ihren Gunsten. Gestiegen ist laut den Studienergebnissen auch der Anteil jener Patienten, die eine Sepsis entwickelten, nämlich von $6,9 \%$ auf $8,5 \%$. Fälle schwerer Sepsis nahmen von 1,7 auf $3,2 \% \mathrm{zu}$. Frauen waren hier ebenfalls rund $20 \%$ häufiger betroffen. Die Mortalitätsraten blieben trotz höherer Sepsisraten über die Zeit stabil bei $0,25 \%$ beziehungsweise 0,20\%. Die Autoren führen dies auf ein verbessertes Management dieser Komplikation zurück.

Unterschiede fanden sich im therapeutischen Vorgehen, die sofortige Dekompression betreffend. 28,6\% der Pa- tienten mussten sich einer solchen Prozedur unterziehen. Dabei geriet im Studienzeitraum die perkutane Nephrostomie (PNS) gegenüber der retrograden ureteralen Katheterisierung weiter ins Hintertreffen. 1999 waren noch $16,1 \%$, 2009 nur noch $11,2 \%$ der entlastenden Eingriffe als PNS vorgenommen worden, die häufiger mit einer Sepsis einherging.

Fazit: Wie mit der Urolithiasis - und besonders im Fall von Infektionen - umzugehen sei, ist dringend weiter zu untersuchen, fordern die Autoren. Sie weisen zugleich darauf hin, dass die von ihnen vorgelegten Beobachtungsdaten nur vorläufigen Charakter haben und der Erzeugung von Hypothesen dienen. Speziell könne nicht auf die Zusammensetzung der Steine geschlossen werden: Wären die Harnsteine von Frauen etwa vor allem vom Struvittyp, könnte die höhere Infektrate schlicht die Tatsache widerspiegeln, dass Frauen häufiger an Harnwegsinfektionen erkranken.

Dr. Robert Bublak

Sammon J et al. Temporal Trends, Practice Patterns, and Treatment Outcomes for Infected Upper Urinary Tract Stones in the United States. Eur Urol 2013; 64: 85-92

\title{
Östrogenantagonist zur Prävention beim Prostatakarzinom?
}

\section{Toremifen ist ein Modulator des Östrogenrezeptors. Eignet er sich auch zur Prostatakarzinomprävention bei Männern mit isolierter hochgradiger prostatischer intraepithelialer Neoplasie?}

E ne hochgradige prostatische intraepitheliale Neoplasie (HGPIN) ist die Vorstufe eines Prostatakarzinoms. Männer mit isolierter HGPIN sind daher gute Kandidaten, um Strategien zur Chemoprävention zu testen. Toremifen ist ein oraler selektiver Östrogenrezeptormodulator, der für die Behandlung von fortgeschrittenem Brustkrebs zugelassen ist. In ersten Studien reduzierte die Substanz auch die Progression einer HGPIN zu einem Prostatakarzinom.

Daher wurde nun in einer über drei Jahre laufenden Phase-III-Studie untersucht, ob Toremifen die Entwicklung eines Prostatakarzinoms bei Männern mit isolierter HGPIN verhindern kann.
1.590 Männer mit HGPIN, aber ohne Prostatakarzinom, erhielten drei Jahre lang randomisiert entweder $20 \mathrm{mg} / \mathrm{d}$ Toremifen oder Placebo. Die Teilnehmer waren 41 bis 87 Jahre alt (median 64 Jahre) und unterzogen sich jährlich einer Biopsie - bis zum Nachweis eines Tumors oder bis zum Studienende.

$34,7 \%$ der Männer in der Placebogruppe und 32,3\% der Männer in der Toremifengruppe entwickelten ein Prostatakarzinom. Das Drei-Jahres-Überleben ohne Prostatakarzinom betrug 54,9\% in der Placebogruppe und 59,5\% unter Toremifen, wobei der Unterschied zwischen beiden Gruppen nicht signifikant war $(\mathrm{p}=0,39)$.
Bei der Analyse verschiedener Variablen wie Rasse, Alter, Prostatakarzinom in der Familienanamnese, Anzahl der Biopsien mit HGPIN, Vorhandensein einer atypischen mikroazinären Proliferation, Prostatagröße und PSA-Wert war in keiner Subgruppe eine Risikoreduktion durch Toremifen nachweisbar. Auch hinsichtlich der Nebenwirkungen und der Todesfälle gab es keine Unterschiede zwischen beiden Studienarmen.

Fazit: Toremifen konnte die Rate der nachgewiesenen Prostatakarzinome nicht verringern. Da Männer mit isoliertem HGPIN ein hohes Risiko für die Entwicklung eines Prostatakarzinoms haben, ist bei diesen Patienten eine Überwachung durch regelmäßige Prostatabiopsien erforderlich.

Dr. Judith Neumaier

Taneja SS et al. Prostate cancer diagnosis among men with isolated high-grade intraepithelial neoplasia enrolled onto a 3-year prospective phase III clinical trial of oral toremifene. J Clin Oncol 2013; 31: 523-9 